Zehua Chen, Maher Mansour, Ulrich Gengenbach, and Liane Koker

\title{
Approaches for Solution-Processed Encapsulation of Printed Medical Wearable Devices
}

https://doi.org/10.1515/cdbme-2020-3034

\begin{abstract}
Wearable medical devices offer a great opportunity to monitor human vital signs to improve healthcare. The use of printing technologies is a promising approach to fabricate the wearables. An encapsulation must be applied to achieve longterm stability and reliability of the printed wearables. In this paper, we discuss the encapsulation requirements of printed wearable medical devices. Different encapsulation approaches are illustrated by means of various examples. Thereby, the focus lies upon solution-processed encapsulation, including the compatible materials and printing technologies.
\end{abstract}

Keywords: encapsulation, wearable devices, printed electronics, medical applications

\section{Introduction}

Wearable medical devices are electronic systems attached to the body, either with direct skin contact or integrated into the clothing, that fulfil medical functionalities. Applications include, but are not limited to, vital signs monitoring, sweat analysis, saliva analysis, wound monitoring, motion/fall detection or magnetic resonance imaging (MRI).

Printing technologies are a promising fabrication process for wearable devices $[1,2]$. The main advantage is the applicability of electronic circuits on surface-conformal substrates that can adapt to the skin, like flexible plastic foils, fabric or paper. The inexpensive substrates and low material consumption due to the additive manufacturing techniques allow for the production of disposable devices, thus avoiding elaborate sterilisation processes. Since a variety of established as well as novel materials can be structured by printing, new functionalities can be realised, especially for sensors. Furthermore, the use of digital printing technologies like inkjet printing or aerosol-jet printing opens up the possibility of manufacturing personalised devices [3]. To realise devices mainly containing electrodes and antennas, the most commonly used technique is

Zehua Chen, Ulrich Gengenbach, Liane Koker, Institute of Automation and Applied Informatics (IAI), Karlsruhe Institute of Technology (KIT), Karlsruhe, Germany, e-mail: zehua.chen@kit.edu

Maher Mansour, German-Jordanian University, Amman, Jordan screen printing due to a high layer thickness and thus a higher conductivity $[4,5]$.

An overview of applications of printed wearable medical devices as well as selected examples are presented in figure 1, including researched approaches and commercial products.

\section{Encapsulation requirements}

For printed wearable medical devices, a conformal encapsulation that protects the printed circuit or components from water (or body fluids like salivary, sweat), oxygen, dirt and dust is required $[15,16]$. The encapsulation methods can be divided into two types, namely, global encapsulation and local encapsulation. A global encapsulation should protect the entire printed structure, while a local encapsulation covers the sensitive parts of the printed structures and leaves parts with sending functions uncovered. It must be biocompatible and should add little volume and weight to the devices. Apart from this, there are some specific requirements, which vary from application to application, e.g for monitoring devices, a selective encapsulation which only allows the analyst to permeate the encapsulation layer is required [17]. Furthermore, it is quite common for wearable devices to work under bending, stretching or twisting forces. Therefore, another requirement of the encapsulation is its flexibility to make sure that even under those forces, the device still works stably [18]. When it comes to commercialization, the cost and the aesthetic appearance of the wearable devices also play an important role for customer's choices. Printed wearable devices should be well designed and fit the customer's taste.

\section{Encapsulation approaches}

Due to the aforementioned encapsulation requirements, flexible encapsulation is used instead of conventional rigid encapsulation like glass or metal [19]. Nowadays, a flexible encapsulation is mostly achieved by barrier foil lamination, thin film encapsulation (TFE) or solution-processed encapsulation. All of them can not only provide high barrier properties but also preserve the flexibility of printed structures [20,21]. 


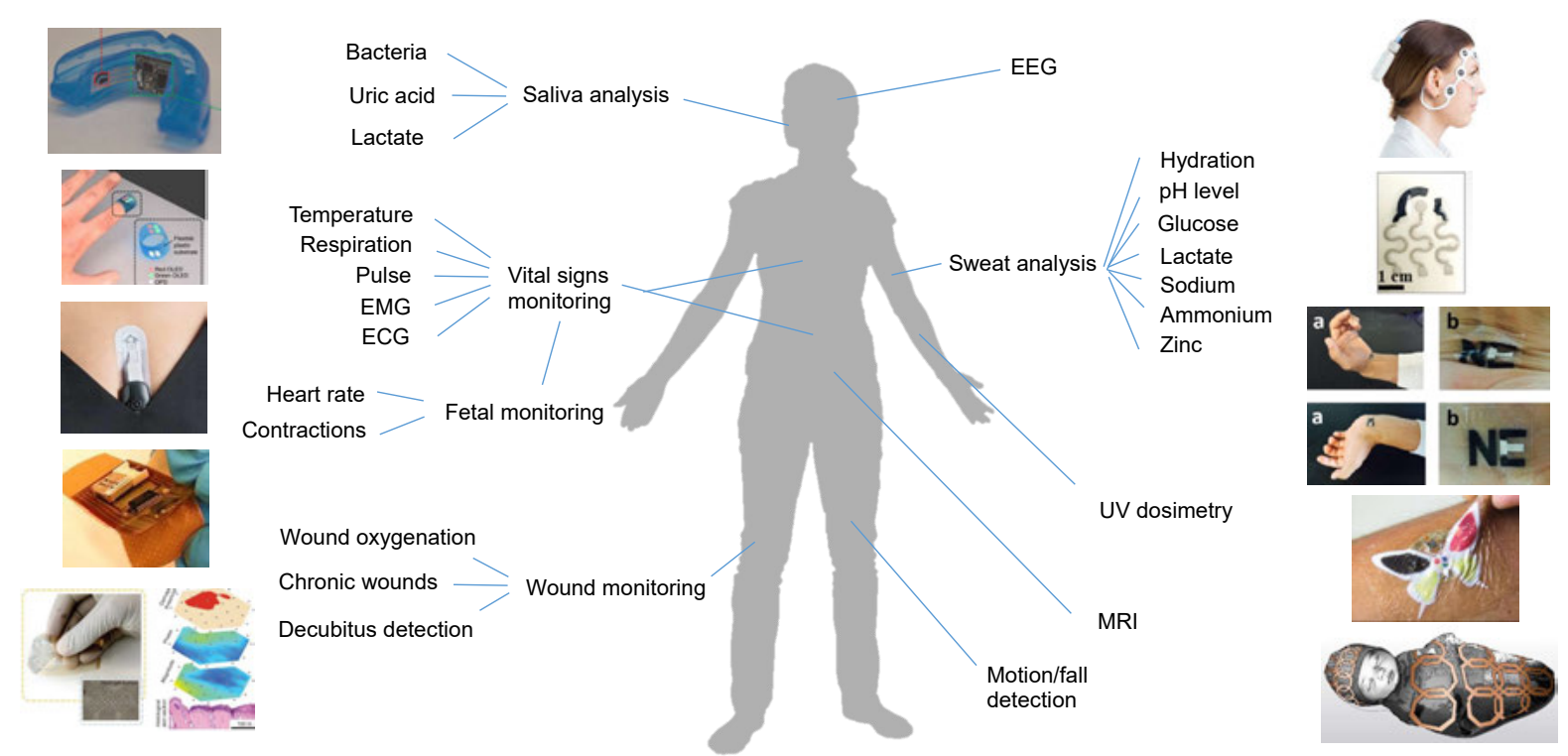

Fig. 1: Overview of applications and selected examples for printed wearable devices, top to bottom left: Screen-printed uric acid mouth-

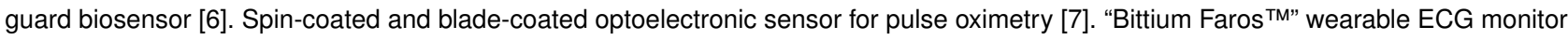
using screen-printed electrodes [8]. Inkjet-printed smart bandage to monitor chronic wounds [9]. Early detection of pressure ulcers by impedance sensing using an inkjet printed electrode array [10]. Top to bottom right: "Bittium BrainStatus ${ }^{\mathrm{TM}}$ " screen-printed disposable EEG electrode set [11]. Stretchable and screen-printed glucose sensor [12] and screen-printed tattoo sensor for monitoring in human sweat [13]. Screen-printed colorimetric UV dosimeter [14]. Screen-printed flexible MRI receiver coils [4]. all figures reprinted with permission.

\subsection{Barrier foil lamination}

In barrier foil lamination, a barrier foil is attached to the substrate mostly by using adhesion, thermal or laser welding. One example is discussed in [22]. Here, an one-step laser encapsulation of a nano-cracking strain sensor was applied. They utilized a laser to cut and bond two polyurethane (PU) films in one step.

\subsection{Thin film encapsulation}

Thin film encapsulation is often used for global encapsulation to protect the whole device. It has been successfully applied to flexible organic light-emitting devices (OLEDs) used for displays as well as wearable devices. E.g. Jeon et al. used multilayer thin film encapsulated flexible OLEDs as light sources in a wearable Photobiomodulation (PBM) patch for skin treatment [23]. In addition, vacuum deposited Parylene coatings are often used in medical devices [17]. Because of the vapor deposition method, Parylene can be applied in very thin layers, but it still provides superior resistance to moisture, solvents. Yokota et al. formed a multilayer consisting of five inorganic (SiON) and organic (Parylene) layers as passivation layer for a smart electronic skin (e-skin) system, the water vapor transmission rates (WVTR) of the passivation layer was approximately $5.0 \times 10^{-4} \mathrm{~g} / \mathrm{m}^{2}$ per day [24].

\subsection{Solution-processed encapsulation}

A drawback of barrier foil lamination is that it cannot offer a conformal encapsulation on a 3D surface. Thin film encapsulation requires additional equipment such as atomic layers deposition (ALD) reactor or vacuum chambers [19]. Thus, we highlight a novel encapsulation approach for printed wearable devices and sensors, that is solution-processed encapsulation. Using this approach, the solvent based, fluid inks or pastes can be applied as encapsulation layer onto printed structures. This allows the encapsulation process to be integrated into the printing process chain. Moreover, both global and local encapsulation can be realised by using different technologies. The methods for solution-processed encapsulation are dropcasting, spin-coating, screen-printing and inkjet-printing, etc.. Drop-casting is a facile coating technique by applying one or more droplets on the target surface [21]. In spin-coating, the solution is deposited and a uniform layer can be formed on a rotating substrate [28]. A low viscosity material is suitable for both drop-casting and spin-coating. These two approaches are usually used for global encapsulation [21]. In screen-printing, an ink with high viscosity is spread over the screen. This approach can not only be applied for global, but also for local encapsulation by using masks. Inkjet-printing is a digital printing process. A low viscosity ink is deposited drop by drop onto the target position. Both global and local encapsulation can be 
Tab. 1: Selected examples for solution-processed encapsulation

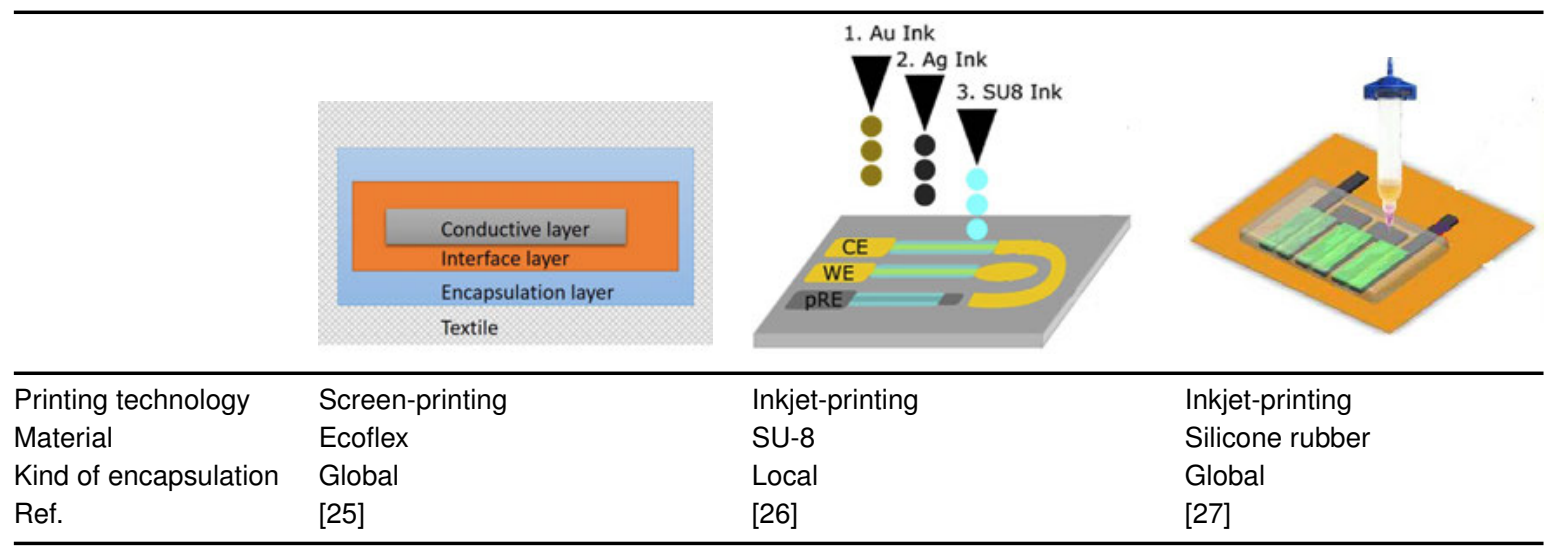

realised without the need for a mask [3, 29]. Some solutionprocessed encapsulation examples are listed in Table 1.

Materials that are compatible with solution-processed encapsulation are fluid inks or pastes such as Ecoflex, SU-8, formulated polymer solutions (PDMS, PI, PVC, etc), dielectric paste $[6,12,25,26,30-36]$. Ecoflex is a printable bioplastic with special properties such as flexibility, toughness and water resistance [37]. SU-8 is a photoresist. The viscosity of SU-8 can be adjusted from $1 \mathrm{cp}$ to $15 \mathrm{cp}$ [38], thus SU-8 inks with different viscosities are compatible with inkjet printing and spin-coating [39]. Polymer solutions with different formulations can reach different viscosities which are compatible with various solution-processed encapsulation methods. Besides, specific functions of an encapsulation layer can be realised, e.g. Hah et al. indicated that by adding $33 \%$ of pure butyl rubber (PIB) into Polydimethylsiloxan (PDMS), a transparent, flexible encapsulant with high moisture stability can be formed [33].

In [35], a bioresorbable polymer solution (poly lactic-coglycolic acid, PLGA) was dropped as an encapsulation layer on top of a screen printed flexible radio frequency (RF) antenna for wireless devices. The PLGA overcoat protects the conductive particles from direct contact to the air and water. Cazalé et al. casted on an inkjet printed electric platform with PVC and dropped the formulated PVC solution to form a $\mathrm{Na}^{+}$-sensitive sealing PVC membrane in a potentiometric microsensor for sweat analysis [34]. In [30], a SU-8 layer was spin-coated on a flexible field effect transistor (FET). The FET can work over 9 days in air and over 65 hours in water stably, which has shown good air stability and water resistance of the SU-8 encapsulation layer. Oh et al. fabricated a PDMS encapsulated transistor, and the PDMS encapsulation layer was formed by spin-coating. This work also indicated that by adjusting the thickness of the encapsulation, a neutral axis position for the conductive layer can be established to maintain the flexibility of the transistor [32]. Khan et al. deposited a thin layer of a fluoropolymer using spin-coating to encapsulate the inkjet printed flexible gold traces. The resistance of the gold traces was monitored via bending to the radius of $5 \mathrm{~mm}$ and $10 \mathrm{~mm}$, and twisting to an angle of $30^{\circ}$. The resistance data remained stable during the bending and twisting test [36]. Kim et al. fabricated a wearable screen printed salivary uric acid mouthguard biosensor and the DuPont 5036 dielectric paste was screen printed on the sensor to confine the electrode areas [6]. In [25], the Ecoflex encapsulation layer was screen printed on the textile strain sensor, which can be used on a glove to detect the finger motion. The strain sensor consists of interface layer, conductive layer and encapsulation layer. All layers were fabricated by screen printing. In this work, waterproof performance of the Ecoflex encapsulation layer was confirmed by demonstrating that after an hour of immersion in water, the LED sample still worked well. Furthermore, in stretching, bending, folding, twisting and dynamic endurance tests, the strain sensors have shown excellent stretchability of $70 \%$. It can be bent to the radius of $1.5 \mathrm{~mm}$, and withstand dynamic stretching and bending endurance tests of 10,000 cycles. Kim et al. demonstrated a wearable temporary screen printed tattoo-based electrochemical sensor for the real-time monitoring of $\mathrm{Zn}^{2+}$ in human perspiration. A transparent insulator was screen printed on the surface of the electrode pattern to confine the electrode and contact areas [13]. Moya et al. demonstrated the fabrication of flexible all-inkjet-printed dissolved oxygen sensors in ambient conditions and without the need for a clean room environment. In this work, a printable SU-8 ink was inkjet printed as passivation layer onto the sensor devices. This all-inkjet-printing approach shorts the processing time and enables rapid prototyping [26]. In [27], a layer of silicone rubber was inkjet printed to cover an entire direct ink written supercapacitor. 


\section{Conclusion}

In this paper, we have discussed the encapsulation requirements of the printed wearable medical devices. Materials and encapsulation approaches such as barrier foil lamination, thin film encapsulation and printable encapsulation were demonstrated. Barrier foil lamination and thin film encapsulation can provide a good protective performance but they have their own drawbacks or limitations. For instance, by using barrier foil lamination, a 3D surface cannot be well encapsulated. For thin film encapsulation, an additional equipment like ALD reactor or a vacuum chamber is needed. Thus, it is concluded that integrating the solution-processed encapsulation with compatible materials into the printing process can be a novel fabrication approach for printed wearable medical devices. Applications were given that illustrate the potential of this encapsulation approach.

\section{References}

[1] S. Khan, S. Ali, and A. Bermak, "Recent Developments in Printing Flexible and Wearable Sensing Electronics for Healthcare Applications," Sensors, vol. 19 , no. 5 , p. 1230,2019

[2] N. Saengchairat, T. Tran, and C.-K. Chua, "A Review: Additive Manufacturing for Active Electronic Components," Virtual and Physical Prototyping, vol. 12, no. 1, pp. 31-46, 2017.

[3] L. Koker, M. Mansour, M. Ungerer, et al., "Potential and Challenges of Inkjet-Printing for Wearable Medical Devices," Biomedical Engineering / Biomedizinische Technik, vol. 64(s2), p. S230, 2019.

[4] J. R. Corea, A. M. Flynn, B. Lechêne, et al., "Screen-Printed Flexible MRI Receive Coils," Nature Communications, vol. 7, no. 1, 2016.

[5] S. Myllymaa, P. Lepola, J. Töyräs, et al., "New Disposable Forehead Electrode Set with Excellent Signal Quality and Imaging Compatibility," Journal of neuroscience methods, vol. 215, no. 1, pp. 103-109, 2013.

[6] J. Kim, S. Imani, W. R. de Araujo, et al., "Wearable Salivary Uric Acid Mouthguard Biosensor with Integrated Wireless Electronics," Biosensors and Bioelectronics, vol. 74, pp. 1061-1068, 2015.

[7] C. M. Lochner, Y. Khan, A. Pierre, et al., "All-Organic Optoelectronic Sensor for Pulse Oximetry," Nature communications, vol. 5, p. 5745, 2014.

[8] Bittium, "Early Detection of Cardiac Abnormalities." product brochure, https://www.bittium.com/medical/bittium-faros\#brochures, 2019.

[9] M. F. Farooqui and A. Shamim, "Low Cost Inkjet Printed Smart Bandage for Wireless Monitoring of Chronic Wounds," Scientific reports, vol. 6 , p. 28949, 2016.

[10] S. L. Swisher, M. C. Lin, A. Liao, et al., "Impedance Sensing Device Enables Early Detection of Pressure Ulcers in Vivo," Nature communications, vol. 6, p. ncomms7575, 2015.

[11] Bittium, "Bittium BrainStatus ${ }^{\mathrm{TM}}$ Wireless EEG Amplifier." https://www. bittium.com/medical/bittium-brainstatus, 2020.

[12] A. Abellán-Llobregat, I. Jeerapan, A. Bandodkar, et al., "A Stretchable and Screen-Printed Electrochemical Sensor for Glucose Determination in Human Perspiration," Biosensors and Bioelectronics, vol. 91, pp. 885-891, 2017.

[13] J. Kim, W. R. de Araujo, I. A. Samek, et al., "Wearable Temporary Tattoo Sensor for Real-Time Trace Metal Monitoring in Human Sweat," Electrochemistry Communications, vol. 51, pp. 41-45, 2015.

[14] H. Araki, J. Kim, S. Zhang, et al., "Materials and Device Designs for an Epidermal UV Colorimetric Dosimeter with Near Field Communication Capabilities," Advanced Functional Materials, vol. 27, no. 2, p. $1604465,2017$.

[15] H. C. Koydemir and A. Ozcan, "Wearable and Implantable Sensors for Biomedical Applications," Annual Review of Analytical Chemistry, vol. 11, no. 1, pp. 127-146, 2018.

[16] M. Ha, S. Lim, and H. Ko, "Wearable and Flexible Sensors for UserInteractive Health-Monitoring Devices," Journal of Materials Chemistry $B$, vol. 6, no. 24, pp. 4043-4064, 2018.
[17] V. Steinmann and L. Moro, "Encapsulation Requirements to Enable Stable Organic Ultra-Thin and Stretchable Devices," Journal of Materials Research, vol. 33, no. 13, pp. 1925-1936, 2018.

[18] K. D. Harris, A. L. Elias, and H.-J. Chung, "Flexible Electronics under Strain: A Review of Mechanical Characterization and Durability Enhancement Strategies," Journal of Materials Science, vol. 51, no. 6, pp. 2771-2805, 2015

[19] D. Yu, Y.-Q. Yang, Z. Chen, et al., "Recent Progress on Thin-Film Encapsulation Technologies for Organic Electronic Devices," Optics Communications, vol. 362, pp. 43-49, 2016.

[20] E. G. Jeong, J. H. Kwon, K. S. Kang, et al., "A Review of Highly Reliable Flexible Encapsulation Technologies towards Rollable and Foldable OLEDs," Journal of Information Display, pp. 1-14, 2019.

[21] M. Eslamian and F. Soltani-Kordshuli, "Development of MultipleDroplet Drop-Casting Method for the Fabrication of Coatings and Thin Solid Films," Journal of Coatings Technology and Research, vol. 15 no. 2, pp. 271-280, 2017

[22] C. Park, H. Jung, H. Lee, et al., "One-Step Laser Encapsulation of Nano-Cracking Strain Sensors," Sensors, vol. 18, no. 8, p. 2673, 2018.

[23] Y. Jeon, H.-R. Choi, M. Lim, et al., "A Wearable Photobiomodulation Patch Using a Flexible Red-Wavelength OLED and Its in Vitro Differential Cell Proliferation Effects," Advanced Materials Technologies, vol. 3, no. 5, p. 1700391, 2018

[24] T. Yokota, P. Zalar, M. Kaltenbrunner, et al., "Ultraflexible Organic Photonic Skin," Science Advances, vol. 2, no. 4, p. e1501856, 2016.

[25] W. J. Lee, J. Y. Park, H. J. Nam, et al., "The Development of a Highly Stretchable, Durable, and Printable Textile Electrode," Textile Research Journal, vol. 89, no. 19-20, pp. 4104-4113, 2019.

[26] A. Moya, E. Sowade, F. J. del Campo, et al., "All-Inkjet-Printed Dissolved Oxygen Sensors on Flexible Plastic Substrates," Organic Electronics, vol. 39, pp. 168-176, 2016.

[27] B. Chen, Y. Jiang, X. Tang, et al., "Fully Packaged Carbon Nanotube Supercapacitors by Direct Ink Writing on Flexible Substrates," ACS Applied Materials \& Interfaces, vol. 9, no. 34, pp. 28433-28440, 2017.

[28] J. Danglad-Flores, S. Eickelmann, and H. Riegler, "Deposition of Polymer Films by Spin Casting: A Quantitative Analysis," Chemical Engineering Science, vol. 179, pp. 257-264, 2018.

[29] Y. Khan, A. Thielens, S. Muin, et al. "A New Frontier of Printed Electronics: Flexible Hybrid Electronics," Advanced Materials, p. 1905279, 2019.

[30] Y.-C. Kung, N. Hosseini, D. Dumcenco, et al., "Air and Water-Stable NType Doping and Encapsulation of Flexible MoS2 Devices with SU8," Advanced Electronic Materials, vol. 5, no. 1, p. 1800492, 2019.

[31] W. Yuan, X. Wu, W. Gu, et al., "Printed Stretchable Circuit on Soft Elastic Substrate for Wearable Application," Journal of Semiconductors, vol. 39, no. 1, p. 015002, 2018.

[32] J. Oh, J.-H. Kim, S. Y. Lee, et al., "Bending Performance of Flexible Organic Thin-Film Transistors with/without Encapsulation Layer," IEEE Transactions on Device and Materials Reliability, vol. 18, no. 1, pp. 14, 2018.

[33] J. Hah, M. Sulkis, C. Ren, et al., "Moisture Barrier, Mechanical, and Thermal Properties of PDMS-PIB Blends for Solar Photovoltaic (PV) Module Encapsulant," in 2019 IEEE 69th Electronic Components and Technology Conference (ECTC), IEEE, 2019.

[34] A. Cazalé, W. Sant, F. Ginot, et al., "Physiological Stress Monitoring Using Sodium Ion Potentiometric Microsensors for Sweat Analysis," Sensors and Actuators B: Chemical, vol. 225, pp. 1-9, 2016.

[35] Y. K. Lee, J. Kim, Y. Kim, et al., "Room Temperature Electrochemical Sintering of $\mathrm{Zn}$ Microparticles and Its Use in Printable Conducting Inks for Bioresorbable Electronics," Advanced Materials, vol. 29, no. 38, p. 1702665, 2017.

[36] Y. Khan, F. J. Pavinatto, M. C. Lin, et al., "Inkjet-Printed Flexible Gold Electrode Arrays for Bioelectronic Interfaces," Advanced Functional Materials, vol. 26, no. 7, pp. 1004-1013, 2015.

[37] BASF, "Ecoflex®: Das Original seit 1998 - zertifiziert kompostierbarer Kunststoff." https://plastics-rubber.basf.com/global/de/performance polymers/products/ecoflex.html. accessed 2020-04-02.

[38] M. Robin, W. Kuai, M. Amela-Cortes, et al., "Epoxy Based Ink as Versatile Material for Inkjet-Printed Devices," ACS Applied Materials \& Interfaces, vol. 7, no. 39, pp. 21975-21984, 2015.

[39] S. J. Moon, M. Robin, K. Wenlin, et al., "Morphological Impact of Insulator on Inkjet-Printed Transistor," Flexible and Printed Electronics, vol. 2, no. 3, p. 035008, 2017. 